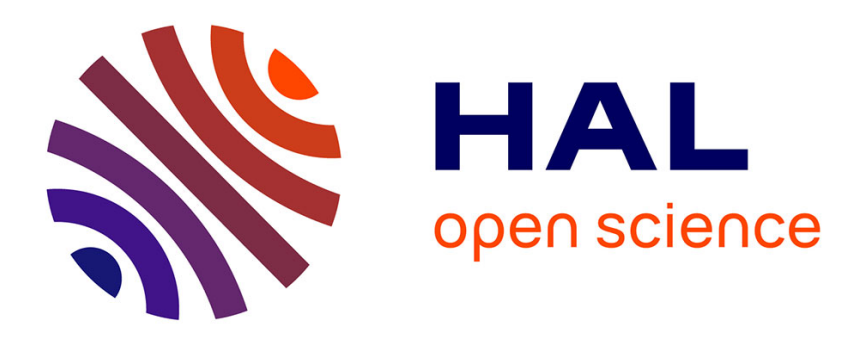

\title{
Lacan avec Heidegger : le dépassement du paradigme linguistique
}

\author{
Stéphane Lojkine
}

\section{To cite this version:}

Stéphane Lojkine. Lacan avec Heidegger : le dépassement du paradigme linguistique. Exil - Transfert - Mémoire / Exil - Transfer - Gedächtnis, Peter Lang, pp.137-148, 2016, 978-3-631-66494-0. hal02495184

\section{HAL Id: hal-02495184 \\ https://hal.science/hal-02495184}

Submitted on 1 Mar 2020

HAL is a multi-disciplinary open access archive for the deposit and dissemination of scientific research documents, whether they are published or not. The documents may come from teaching and research institutions in France or abroad, or from public or private research centers.
L'archive ouverte pluridisciplinaire HAL, est destinée au dépôt et à la diffusion de documents scientifiques de niveau recherche, publiés ou non, émanant des établissements d'enseignement et de recherche français ou étrangers, des laboratoires publics ou privés. 


\title{
Lacan avec Heidegger : le dépassement du paradigme linguistique
}

\author{
Stéphane Lojkine (Aix-Marseille Université)
}

Lacan a fait partie des premiers lecteurs français de Heidegger. Il l'a peut-être découvert grâce au cours donné par Jean Wahl ${ }^{1}$ en Sorbonne de janvier à juin 1946: Jean Wahl y condensait ses notes sur le séminaire de Heidegger suivi à Fribourg en 1928; ${ }^{2}$ peut-être aussi à l'occasion des quatre conférences d'Emmanuel Lévinas sur Le Temps et l'Autre, données en 1946-1947 au Collège philosophique.

Lacan a voulu rencontrer Heidegger, l'a rencontré finalement. Lorsque Heidegger se rend en France, en 1955, pour ainsi dire incognito, à l'instigation de Jean Beaufret et de Maurice de Gandillac, pour prononcer sa conférence «Qu'est-ce que la philosophie ? », Lacan, qui s'était rendu à Fribourg quelques mois plus tôt, le reçoit dans sa gentilhommière de Guitrancourt : mais hormis quelques promenades et des conversations insignifiantes, ils n'ont pas d'échange intellectuel sérieux. ${ }^{3}$

Que cherchait Lacan ? Heidegger apparaît dans la pensée lacanienne au moment où ce dernier se livre à une modélisation linguistique des théories de Freud. Mais Heidegger ne sert pas cette modélisation : tout au contraire, il ouvre à Lacan la possibilité d'une résistance à la pensée structurale de la langue, et de son dépassement par la déconstruction de la notion même de structure. Heidegger avait fait paraître en 1951 un article intitulé « Logos » dans un recueil de mélanges en l'honneur de son collègue Hans Jantzen. Lacan traduit cet article en français durant l'année 1955 : il paraît en 1956 dans le numéro 1 de la revue La Psychanalyse .4

\footnotetext{
${ }^{1}$ Lacan se réfère à « quelques articles que M. Jean Wahl a consacrés récemment [aux textes de Heidegger sur le Dasein] » dans la séance du 27 juin 1956 du Séminaire (Lacan, Jacques: Séminaire III, Les Psychoses. Seuil: Paris 1981, p. 339)

${ }^{2}$ Voir Janicaud, Dominique: Heidegger en France. Abin Michel: Paris 2001, vol. 1, p 94-97.

${ }^{3}$ Janicaud, Dominique: op. cit., p. 149-150.

4 Heidegger, Martin: «Logos», trad. J. Lacan. La Psychanalyse: revue de la société française de psychanalyse. $1956, \mathrm{n}^{\circ} 1, \mathrm{p} .59-79$.
} 


\section{I. «Logos » de Heidegger}

\section{Le logos comme dispositif d'accueil de la parole}

Hans Jantzen était historien de l'art, spécialiste de l'architecture gothique. Il avait étudié notamment ce qu'il appelait la structure diaphane du cloisonnement gothique ${ }^{5}$, un concept désormais fondateur pour l'architecture contemporaine. Il n'est pas indifférent que la déconstruction à laquelle Heidegger se livre de la notion de logos, à partir d'une analyse lexicologique de l'aphorisme d'Héraclite sur l'Ev $\pi \dot{\alpha} \nu \tau \alpha$ vienne en quelque sorte symboliquement s'inscrire dans la structure diaphane de Jantzen. Heidegger procède ici de la même manière qu'il l'avait fait quelques années plus tôt avec la parole d'Anaximandre ${ }^{6}$, la plus ancienne parole philosophique connue : en restituant aux termes qui sont devenus, dans la philosophie post-platonicienne, des catégories abstraites et logiques de pensée, leur matérialité pratique, leur spatialité et leur plasticité originelles. Le discours grec, disséminé en disposition d'un dévolu et d'un recueilli, en installation d'un mandat qui fait événement, s'organise, au fil de la démonstration heideggérienne, en une mise en espace, un agencement qui précède la parole, se prépare à l'accueillir. Cette disposition, cet agencement se substituent à l'articulation au signifié, qui devient secondaire, postérieure. Le problème du signifié est en quelque sorte évacué par Heidegger au profit du dispositif qui accueille la parole, selon une configuration qui n'est pas sans faire écho à la structure diaphane de l'architecture gothique :

« Ainsi ce qui parle dans le langage en étant au niveau du $\lambda \dot{\varepsilon} \gamma \varepsilon เ \nu$ comme fait de mettre-à-reposer, ne trouve à se déterminer ni à partir de la vocalisation $(\phi \omega \nu \eta \dot{\eta})$, ni à partir du fait de signifier

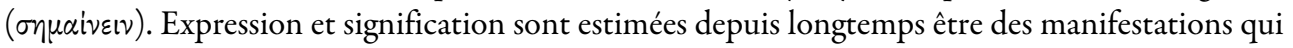
offrent les traits indubitables du langage. Mais elles ne touchent pas proprement au domaine marqué de l'empreinte originelle qui est l'essence du langage, non plus qu'elles ne peuvent généralement déterminer ce domaine dans ses traits principaux. $\gg^{7}$

Heidegger définit ici nettement sa démarche comme ontologique : c'est-à-dire qu'elle vise à dégager un «domaine » du logos, à définir dans ce domaine une «empreinte originelle », à inférer de cette empreinte «l'essence du langage ». A cette démarche

5 Jantzen, Hans: «Sur l'espace intérieur de l'église gothique » (1927). In: Beaufret, Jean: Leçons de philosophie, I. Introduction, libres propos au sujet de « Qu'est-ce que la philosophie ? », Seuil Traces écrites: Paris 1998, p. 20-21.

${ }^{6}$ Heidegger, Martin: «La parole d'Anaximandre » (1946). In: Id: Chemins qui ne mènent nulle part. Gallimard: Paris 1962, Tel, 2012, p. 387 sq.

${ }^{7}$ Je cite Heidegger dans la traduction de Lacan. Heidegger, Martin: «Logos », op. cit., p. 65. Voir également Resweber, Jean-Paul: « De la traduction à l'interprétation ». In dossier « Traduire Heidegger - Lacan », Le Portique, vol. 31 (2013), p. 27-71. 
ontologique, déconcertante à première vue, il oppose l'approche grammaticale classique, qui va produire, motiver la démarche structurale: de l'opposition grecque entre $\phi \omega \nu \varepsilon \tilde{\sigma} \theta \alpha \iota$ et

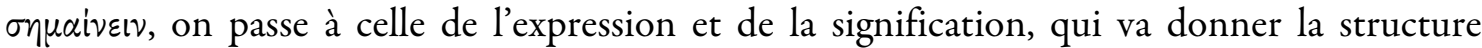
binaire du signe chez Saussure, séparé en un signifiant et un signifié. Si Heidegger tait ce dernier avatar, c'est pourtant bien contre lui qu'il opère cette déconstruction ontologique du logos grec.

\section{La déconstruction heideggérienne : dispositif et ontologie}

Il s'agit donc de repartir du sens pré-discursif du verbe grec $\lambda \dot{\varepsilon} \gamma \varepsilon \iota \nu$, récolter, ramasser, et de faire travailler ce sens. Lacan traducteur s'ingénie à rendre la mise en travail heideggérienne de la langue allemande : porter à gésir, mettre à reposer, jeter le dévolu. On peut douter que $\lambda \varepsilon \dot{\gamma} \varepsilon\llcorner\nu$ ait jamais techniquement signifié tant de choses, mais il ne s'agit pas de cela. Heidegger convertit la phrase d'Héraclite en un tableau, un contexte, une image dans laquelle repérer une empreinte ontologique :

«La mise en réserve de ce qui doit être rentré a déjà marqué sa prise sur les démarches de la moisson dès leur départ et sur leur ensemble dans l'intrication de leur suite. Nous fixons-nous seulement sur le tour à tour de ces démarches, alors au relevage et à l'émondage succède le ramassage, à celui-ci la rentrée de la récolte, à celle-ci la mise à l'abri dans des récipients et des resserres. $\gg^{8}$

On aurait tort de réduire ce qui s'exprime ici à un simple travail de la métaphore, à moins d'entendre métaphore littéralement, comme transfert, et ici transfert de la linéarité logique du discours (le logos post-platonicien), vers la plasticité d'un tableau vivant se déployant dans le temps. Dans le tableau, quelque chose «marque sa prise », imprime son empreinte ontologique : ce quelque chose, c'est «la mise en réserve », le legein, qui est, seul, inintelligible, mais prend sens dans le tableau général. Dans le travail des saisons, dans l'enchaînement des travaux des champs, se manifeste la forme dynamique du legein; il s'agit toujours de mettre en réserve; mettre en réserve est l'essence à quoi toutes ces activités se ramènent.

A ce moment là de l'exposé, le logos est totalement déconstruit : la catégorie logique du discours a complètement disparu, la vérité essentielle du logos s'est disséminée dans les travaux

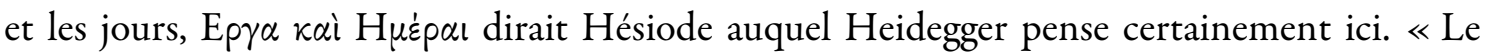

\footnotetext{
8 Heidegger, Martin: « Logos », op. cit., p. 62.
} 
point de remembrement où nous en sommes parvenus contient déjà la réponse », écrit-il alors. Ce point de remembrement, c'est précisément le changement de logique auquel le transfert du discours vers le tableau nous conduit. Il ne s'agit plus de repérer, étymologiquement ou historiquement, comment un concept comme celui de logos a changé de sens, et par exemple de définir un logos pré-socratique auquel le discours logique aurait succédé, mais d'introduire dans le tableau du logos hésiodique ou héraclitéen un événement qui sera l'événement de l'avènement du langage. Ce ne sont pas deux conceptions sémantiquement différentes qui se succèdent dans une catégorie logique techniquement immuable; c'est un seul dispositif qui se construit, d'abord comme assise spatiale du « mettre en réserve », puis, dans cette assise (c'est-à-dire ni à côté d'elle, ni pour la remplacer), comme événement de la parole.

« Nous n'avons nullement eu à faire dans ce qui précède avec la signification mouvante des mots, mais bien plutôt sommes-nous venus buter contre un véritable événement dont le caractère peu rassurant se dissimule encore dans la simplicité qui l’a fait jusqu’à présent passer inaperçu. »

Comment caractériser cet événement de la parole que nous ne percevons plus aujourd'hui comme événement, dont la simplicité même nous dissimule l'inquiétante étrangeté ? C'est ici que, subrepticement, transpire la structure diaphane du cloisonnement gothique de Jantzen. Quelque chose est occulté, l'événement essentiel de la parole est occulté, mais il est occulté par du diaphane, par l'évidente simplicité de ce qui a l'apparence d'un nonévénement.

\section{Contamination du logos : de la syntaxe à la fractale}

Heidegger repart ici de l'ensemble de l'aphorisme d'Héraclite, pour démontrer que le « mettre en réserve » consubstantiel au $\lambda \dot{\varepsilon} \gamma \varepsilon \iota \nu$ s'y contamine dans toute la phrase et s'y déploie comme dispositif antécédant la parole. L'aphorisme est le suivant :

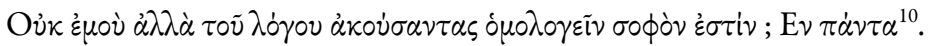

On pourrait traduire, naïvement sans doute, comme suit : Ce n'est pas moi, mais le logos qu'il s'agit d'écouter pour convenir que l'Un est toutes choses. Autrement dit: ce n'est pas

9 Heidegger, Martin: « Logos », op. cit., p. 64.

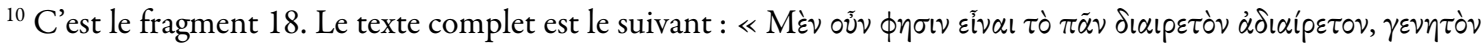

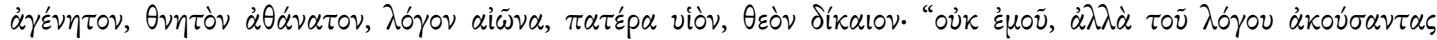
ó comme d'Héraclite. 
parce que c'est moi Héraclite qui le dis, mais c'est la logique même du logos qui le veut; il est sage de convenir que l'Un est toute chose.

Heidegger fait remarquer qu'il ne s'agit pas simplement ici d'une parole qui s'exprime, même double : d'un côté la parole d'Héraclite, de l'autre le principe même de toute parole.

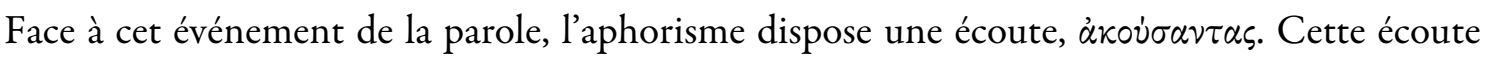
n'est pas une simple réception, mais une acceptation, une compréhension, autrement dit à nouveau un « mettre en réserve ». Puis, entre les auditeurs qui écoutent, il installe un accord,

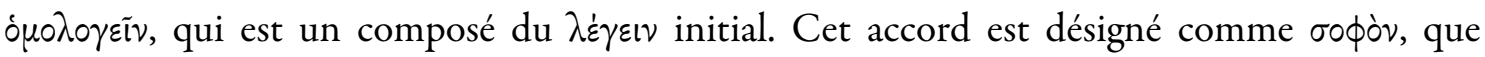
Heidegger ne traduit pas par « sage » selon l'acception classique, mais par « mandaté » :

«Voici donc comment vient à être ce qui tient du mandaté, quand les mortels accomplissent ce

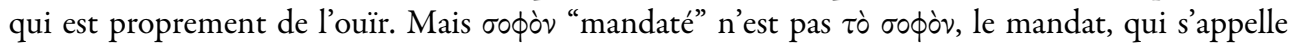
ainsi parce qu'il répartit en soi tout ce qui est destin, et précisément aussi ce qui s'en fait voir dans la destination du maintien mortel. [...] Et déjà nous sommes arrêtés devant la nouvelle énigme

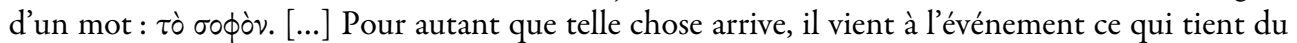

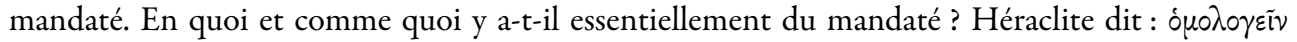

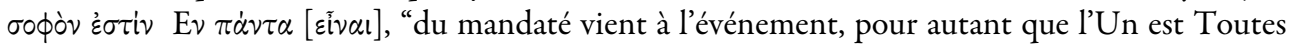
Choses”. ${ }^{11}$

A ce moment de la progression heideggérienne, à laquelle la traduction lacanienne ajoute peut-être un tour de plus d'obscurité, le lecteur a de quoi être désarçonné. Il s'agissait $a$

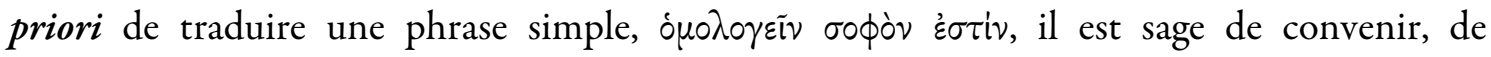
s'accorder, de tomber d'accord que. Mais la forme originelle du $\lambda \dot{\varepsilon} \gamma \varepsilon \iota \nu$ initial, cette empreinte, cette figure de la mise en réserve s'étend métonymiquement, contamine le $\sigma o \phi o ̀ v$, qui désigne à son tour «comment vient à être ce qui tient du mandaté », ce qui est une manière d'interroger cette réserve dont le logos introduit le geste, ou plus exactement à la fois l'espace et l'événement. Heidegger déploie cette forme originelle grâce au mouvement de la parole vers l'écoute, «ce qui est proprement de l'ouïr », le fait d'accueillir ce qui est mandaté, dévolu, moissonné, recueilli. Le déploiement de la phrase n’obéit donc pas à une logique syntaxique, mais plutôt à un principe récursif : c'est un déploiement gigogne, une organisation fractale de l'objet, dont la formule est le logos liminaire indéfiniment repris : chaque profération, chaque « mandat » s'inscrit dans un accueil qui est lui-même proféré depuis un domaine d'accueil qui... etc. Du noyau originel, ce logos qu'il s'agit d'écouter, on passe à l'accueil du logos, qui

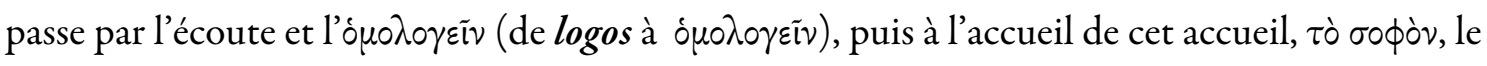
mandat, puis à ce que mandate le mandat, le contenu de la parole accueillie dans l'accueil de

11 Heidegger, Martin: « Logos », op. cit., p. 70-71. 
cet accueil, Ev $\pi \dot{\alpha} \nu \tau \alpha$, où l'on retrouve l'événement de la parole, l'Un, l' Ev, et la dissémination de la moisson préalable à ce recueillement, à cette dévolution, à ce mandat, les $\pi \dot{\alpha} \nu \tau \alpha$, Toutes choses.

Le passage de la syntaxe à la fractale, ou autrement dit du discours à une structure diaphane du cloisonnement, isole un noyau essentiel, atemporel, désigné comme le «mandaté», le dévolu, comme ce qui vient à être, autour duquel se disposent concentriquement les enveloppes conjoncturelles, mortelles, du recueillement, de l'ouïr, du dépôt, de la moisson, inscrites dans le temps et l'événement («il vient à l'événement », « du mandaté vient à l'événement »). C'est pourquoi on voit apparaître ce terme de mortel qui n'apparaissait pas dans l'aphorisme originel d'Héraclite : « quand les mortels accomplissent ce qui est proprement de l'ouïr », «ce qui s'en fait voir dans la destination du maintien mortel $\gg$.

\section{L'être comme noyau insaisissable}

Mais un noyau essentiel est-il réellement isolé ? c'est l'objet de la dernière partie de l'article, la plus difficile. La forme métonymique ultime de l'empreinte initiale, le « mettre en réserve » du logos, est la formule Ev $\pi \dot{\alpha} \nu \tau \alpha$, qu'on traduit «l'Un est toutes choses », en suppléant la copule عĩval, le verbe être comme articulation syntaxique entre Ev le sujet et $\pi \dot{\alpha} \nu \tau \alpha$ son attribut. Or justement tout le remembrement heideggérien vise ici à isoler ce verbe être non comme un simple outil verbal qu'on peut aisément élider, mais comme une sorte de copule primordiale où se jouerait le mystère originel de l'être.

Or, nous dit Heidegger, ce n'est pas le style d'Héraclite :

«Le Ev $\pi \dot{\alpha} v \tau \alpha$ suffit. Mais il n'est pas seulement suffisant. Il demeure pour soi beaucoup plus conforme à la chose ici pensée et aussi au style de ce que dit Héraclite : E $\nu \pi \dot{\alpha} \nu \tau \alpha$, l'Un-Toutes choses, Tout-l'Un.

Comment à les dire prendre ces mots légèrement ? Quel éclaircissement s'en donner à les proférer dans l'approximatif ? Une diversité embrouillée de significations trouve à se nicher dans les deux mots dangereusement inoffensifs $E v$ et $\pi \dot{\alpha} \nu \tau \alpha$. L'indétermination de leurs nœuds donne licence à des propositions à plusieurs sens. $\gg^{12}$

Une fois encore, le problème de l' Ev $\pi \dot{\alpha} \nu \tau \alpha$ ne se résout pas en termes de signification, de ce qu'il s'agit de signifier, et qui ne conduit qu'à des approximations et à des égarements ; l' Ev $\pi \dot{\alpha} \nu \tau \alpha$ doit être saisi comme disposition de l'Être, c'est-à-dire comme une configuration

${ }^{12}$ Heidegger, Martin: « Logos », op. cit., p. 71. 
dans l'espace prête à accueillir son événement. L'effet de signification brouille le principe de configuration. Heidegger mime ce brouillage ou cette ivresse par un jeu de consonances, dont Lacan s'est plu à trouver des équivalents français. Le pur jeu du signifiant force l'abolition de la syntaxe et installe la scène de l'avènement ontologique :

« Ev est l'uniquement-Un en tant que ce qui unit. Il unit en même temps qu'il répartit. En même temps qu'en lisant ce qu'il élit, il laisse se présenter ce qui se présente comme tel, il le répartit en son entier ? L'uniquement-Un unit en tant qu'il est le lais où se lit ce qui s'élit. Ce fait ici d'unir ce qui dans ce lit se lit, réparti en soit ce qui unit jusqu'au point qu'il est cet un-ci, et en tant qu'il est cet un-ci, aussi l'Unique. Le Ev $\pi \dot{\alpha} \nu \tau \alpha$ qui est nommé dans la parole d'Héraclite nous ouvre un simple cillement sur ce qu'est le Logos. $\gg^{13}$

Dans cette glossolalie de l' Ev $\pi \dot{\alpha} \nu \tau \alpha$ reviennent les verbes unir, répartir, se présenter. Heidegger superpose le geste du rassemblement et celui de la séparation, ce qu'il en est de l'articulation logique du logos, et sa désarticulation, sa déconstruction disséminante. La phrase s'ordonne autour de la formule incantatoire « le lais où se lit ce qui s'élit », qui désigne la copule nodale autour de laquelle s'organise le mandat et le recueillement, mais qui ne la désigne qu'en l'élidant : l'être disparaît de l'énoncé, qui se constitue comme son enveloppe. Non pas directement l'être, mais un chant pour le dire, le lais, un chant qui est aussi un espace de recueillement, lais comme ce qui est laissé, un chant dont le contenu, l'objet d'élection, « ce qui s'élit », n'est indiqué qu'allusivement, comme ce qui est donné à lire mais ne peut être proféré.

\section{Heidegger dans Le Séminaire de Lacan}

Pour comprendre le rôle que va jouer cette référence heideggérienne dans la pensée de Lacan, il faut remonter un peu en arrière, au livre I du Séminaire, à l'année 1953-1954, consacrée aux écrits techniques de Freud. Sur les 24 séminaires de Lacan, 16 contiennent au moins une référence à Heidegger, souvent beaucoup plus. La première référence surgit à la faveur de la discussion qui clôt la séance du 19 mai 1954, consacrée au noyau du refoulement. Lacan y développe une thèse qui était déjà en germe dans ses tout premiers articles d'avantguerre consacrés à la psychose : il n'y a pas d'événement objectif du trauma dont les troubles psychiques ultérieurs seraient la conséquence. Reprenant l'analyse de L'Homme au loup, où Freud développe sa thèse de la scène primitive, il montre que c'est la névrose qui construit $a$ posteriori le trauma originaire dont elle se nourrit, même si le trauma a bien une existence

13 Heidegger, Martin: « Logos », op. cit., p. 72. 
réelle et ne constitue jamais une pure affabulation. La névrose est donc le premier travail analytique, et son origine est un futur antérieur (la névrose aura eu, aura construit sa propre origine). Du coup, le refoulement, dont le processus constitue la névrose, et le retour du refoulé - dans le rêve, les actes manqués, les lapsus qui constituent le matériau de l'analyse psychanalytique - sont une même chose. Paradoxalement, le retour du refoulé crée le refoulement en même temps qu'il en découle. Peut-on, dès lors, simplement et heureusement, oublier, interroge Olivier Mannoni ? Réponse de Lacan :

« Oui, sans retour du refoulé. L'intégration dans l'histoire comporte évidemment l'oubli d'un monde entier d'ombre qui ne sont pas portées à l'existence symbolique. Et si cette existence symbolique est réussie et pleinement assumée par le sujet, elle ne laisse aucun poids derrière elle. Il faudrait faire alors intervenir les notions heideggeriennes. Il y a dans toute entrée de l'être dans son habitation de paroles une marge d'oubli, un $\lambda \dot{\eta} \theta \eta$ complémentaire de toute $\dot{\alpha} \lambda \dot{\eta} \theta \varepsilon 1 \alpha . \gg^{14}$

Lacan ne se réfere pas ici nécessairement à l'article «Logos» de Heidegger, car la méditation sur $\dot{\alpha} \lambda \dot{\eta} \theta \varepsilon ı \alpha$ remonte à la fin de la première section d'Âtre et Temps ${ }^{15}$ (1927), est reprise dans De l'essence de la vérité (1931-1932) et dans les Questions II (1947). Mais il a pu trouver aussi dans « Logos » les formulations auxquelles il se réfère ici :

«Le fait de révéler a besoin du voilement. L' $2 \lambda \eta \dot{\eta} \theta \varepsilon 1 \alpha$ repose dans le Léthé, puise en lui, produit ce qui par son travers est relégué. Le logos est en soi à la fois une révélation et un recel. Il est l' $\dot{\alpha} \lambda \dot{\eta} \theta \varepsilon 1 \alpha$. Le dévoilement a besoin du voilement du Léthé, comme de la réserve dans laquelle la révélation puisse en quelque sorte puiser. Le Logos, le lais où se lit ce qui s'élit, a en soi le caractère de ce qui sauvegarde en révélant. $»^{16}$

La question de la vérité est intimement liée à celle du logos, et c'est dans cette liaison qu'intervient Heidegger pour l'élaboration lacanienne. Il s'agit toujours de ce mouvement de mise en réserve, que Lacan lit chez Heidegger comme le mouvement constitutif de l'inconscient: c'est dans ce mouvement que se manifeste la vérité, et c'est dans ce même mouvement qu'advient le logos, qu'il ne faut pas trop se précipiter à définir comme le langage, ni même comme la parole, car en lui l'empreinte essentielle est celle d'un mouvement dans un espace, c'est-à-dire l'installation d'un dispositif. Dans ce mouvement, la «marge d'oubli » (Lacan), le « voilement » (Heidegger) jouent un rôle essentiel que la « structure diaphane »

14 Lacan, Jacques: Le Séminaire, livre I, Les Écrits techniques de Freud, 1953-1954, Séance du 19 mai 1954, « Le noyau du refoulement ». Seuil: Paris, 1975, p. 216.

${ }^{15}$ Heidegger, Martin: Etre et temps, lère section, $6^{e}$ chapitre, $\$ 44$, «Dasein, ouvertude et vérité », b) « Le phénomène original de la vérité et ses soubassements ontologiques », p. 219-220 (édition de Tübingen, 1927), Gallimard: Paris 1986, p. 270-271. p.219-220: original allemand? OUI

16 Heidegger, Martin: « Logos », op. cit., p. 73. 
de Jantzen permet d'imager. Lacan parlera, au Séminaire IV sur la relation d'objet, de fonction du voile ${ }^{17}$.

Lacan a l'air ici de concéder à Mannoni la possibilité d'un oubli pur, en dehors du mécanisme du refoulement et du retour du refoulé, mais il le fait en se référant à Heidegger, pour qui l'oubli n'est pas une suppression, mais au contraire la manifestation du voile, c'est-àdire l'installation du dispositif ontologique. L'oubli lacanien vient ici en plus de l'appareil psychanalytique traditionnel, et en marge de lui, comme possibilité d'une réussite de l'être, comme éventualité du bonheur, comme affirmation qu'il existe quelque chose en dehors de la névrose. Mais ce dehors, ce supplément gratuit évoqué en fin de séance contient en fait le germe de toute la modélisation lacanienne à partir de laquelle seront repensés le refoulement, la constitution du sujet et l'articulation de ces processus à une pensée structuraliste du langage.

Car si l'on ne devait retenir qu'une formule de la pensée lacanienne, c'est bien celle de l'inconscient structuré comme un langage, formule trop souvent mal interprétée précisément parce qu'elle néglige le transfert heideggerien qui en constitue le soubassement : ce langage qui a l'air d'être celui de Saussure, qui emprunte à la linguistique ses signes et ses mots, le $S$ du signifiant et du sujet, le s du signifié et de l'inconscient, la barre sémiotique comme coupure de la castration symbolique, ce langage qui semble structurer la production lacanienne des mathèmes est aussi le logos heideggerien, comme en témoigne, dès le livre III du Séminaire, cette mise au point qui vaut mise en garde :

«Le signifié, ce ne sont pas les choses toutes brutes, déjà là données dans un ordre ouvert à la signification. La signification, c'est le discours humain en tant qu'il renvoie toujours à une autre signification. M. Saussure dans ses célèbres cours de linguistique, représente un schéma avec un flux qui est la signification, et un autre, qui est le discours, ce que nous entendons. [...] Ce schéma est discutable. [...] Un système du signifiant, une langue, a certaines particularités qui spécifient les syllabes, les emplois des mots, les locutions dans lesquelles ils se groupent, et cela conditionne, jusque dans sa trame la plus originelle, ce qui se passe dans l'inconscient $\gg .{ }^{18}$

Par cette récusation du double flux saussurien se prépare ici ce qui sera formulé plus nettement encore dans «L'instance de la lettre » : l'évacuation du signifié au profit d'une théorisation du langage comme pur «système du signifiant », dans lequel le signifié n'est

17 «Il s'agit ici de la descente sur le plan imaginaire du rythme ternaire sujet - objet - au-delà, qui est fondamental de la relation symbolique. Autrement dit, dans la fonction du voile, il s'agit de la projection de la position intermédiaire de l'objet. » (Lacan, Jacques: Le Séminaire, livre IV, La Relation d'objet, 1956-1957, Séance du 30 janvier 1957, « La fonction du voile ». Seuil: Paris, 1994, p. 157.)

${ }^{18}$ Lacan, Jacques: Le Séminaire, livre III, Les Psychoses, 1955-1956, Séance du $1^{\text {er }}$ février 1956, « Du non-sens et de la structure de Dieu ». Seuil: Paris 1981, p. 135. 
qu'un signifiant positionné autrement, tombé dans l'inconscient, refoulé sous la barre sémiotique. On voit ici comment «le signifié » est d'abord dilué dans «la signification », puis disparaît au profit d'un « système du signifiant ». Le signifiant même change de nature, cesse d'être pensé comme une entité isolable: «Le discours humain en tant qu'il renvoie toujours à une autre signification » donnera naissance à la notion de chaîne signifiante, par laquelle Lacan sort d'une modélisation strictement linguistique, ou tout du moins strictement saussurienne, pour penser un espace d'accueil, de mise en réserve, l'inconscient, où vient se tramer, se disposer, s'organiser quelque chose qui n'est pas une entité nommable, mais bien plutôt le jeu articulatoire de la copule, par quoi se définit chez Heidegger l'être :

« Ce n'est pas dire que le symptôme est toujours fondé sur un calembour, mais il est toujours fondé sur l'existence du signifiant comme tel, sur un rapport complexe de totalité à totalité, ou plus exactement de système entier à système entier, d'univers du signifiant à univers du signifiant. $\gg^{19}$

Le signifiant devient rapport, et rapport «de totalité à totalité », c'est-à-dire irréductible à un mécano structural. Ces réflexions surviennent à l'occasion d'une analyse des «Remarques psychanalytiques sur l'autobiographie d'un cas de paranoïa: le président Schreber $\gg^{20}$, dans laquelle Lacan cherche à établir la validité du document que constituent les Mémoires de Schreber ${ }^{21}$ pour comprendre la logique et le processus de sa psychose. Le délire des Mémoires ne peut pas être traité comme articulation d'un flux du discours à un flux de signification, et fait précisément symptôme parce qu'il est travaillé par le non-sens. Ce serait également une erreur que de supposer une rationalité du délire antérieure au langage, dont le discours du psychotique ne serait que le fragment défiguré, le pâle reflet. Il faut prendre ces Mémoires comme une totalité signifiante, comme un système entier, comme un univers du signifiant qui s'articule à un autre système entier, un autre univers du signifiant, celui de la psychose même de Schreber.

Évidemment ce principe de méthode, initié par Freud, dépasse largement la sphère de la psychanalyse, parce qu'il induit une conception globale de la nature du langage. Lacan en est pleinement conscient, et convoque pour cette raison précisément Heidegger :

« D'abord, y a-t-il un interlocuteur?

\footnotetext{
19 Ibid.

${ }^{20}$ Freud, Sigmund: Le Président Schreber : un cas de paranoïa, 1911, trad. française O. Mannoni, Payot Poche: Paris 2011.

${ }^{21}$ Schreber, Daniel-Paul: Mémoires d'un névropathe, 1903, Seuil Points Essais: Paris 1985.
} 
Oui, il y en a un, et qui dans son fond est unique. Cette Einheit est très amusante à considérer, si nous pensons à ce texte de Heidegger sur le logos que j’ai traduit, que vous allez voir paraître dans le premier numéro de notre nouvelle revue, La Psychanalyse, et qui identifie le logos avec le En héraclitéen. Et précisément, nous verrons que le délire de Schreber est à sa façon un mode de rapport du sujet à l'ensemble du langage. $\gg^{22}$

Le monologue du président Schreber, même délirant, est adressé, il a un interlocuteur. Mais cet interlocuteur n'est pas une personne, c'est «l'ensemble du langage ». Ses Mémoires nous confrontent par là à ce qu'il en est globalement du langage, comme système, comme mode articulatoire, comme principe de fonctionnement. Ce système, ce bloc, cette Einheit, Lacan les réfère au $\mathrm{E} v \pi \dot{\alpha} \nu \tau \alpha$ d'Héraclite, que Heidegger identifie au nœud essentiel de l'être depuis lequel penser le logos. Cela veut dire qu'au moment où il généralise le paradigme linguistique comme instrument d'une exégèse rationalisée des écrits de Freud, Lacan subvertit radicalement ce paradigme, en faisant basculer la sémiotique saussurienne vers une logique de la configuration, du dispositif où le langage est compris comme logos héraclitéen, comme principe d'articulation, de voilement et de dévoilement, de disposition.

Le 13 juin 1956, au cours d'une séance du même séminaire, Lacan fait à nouveau référence à Heidegger pour imager, à la manière du maître de Fribourg, ce dispositif :

«Bref, je voudrais vous amener à une répartition des fonctions du langage autre que ces ânonnements autour de la locution, de la délocution et de l'allocution. Et ce, en fonction de la question, la question toujours latente, jamais posée.

Si elle vient au jour, si elle surgit, c'est toujours en raison d'un mode d'apparition de la parole que nous pouvons appeler de différentes façons, la mission, le mandat, la délégation, ou encore la dévolution, par référence à Heidegger. C'est le fondement, ou la parole fondatrice - Tu es ceci, ma femme, mon maître, mille autres choses. Ce tu es ceci, quand je le reçois, me fait dans la parole autre que je ne suis $\gg .^{23}$

Cette question latente, que le sujet se pose et où se joue sa constitution essentielle, est la question que la psychanalyse se fixe comme objet. Mais elle est aussi l'interrogation heideggerienne de l'être : le mandat, par quoi Heidegger définit le logos héraclitéen, rencontre ici heureusement la mission que Schreber prétend lui avoir été assignée par les anges. Cette rencontre ne fait pas de Lacan un heideggerien : elle facilite le dépassement qu'il initie du paradigme linguistique vers la logique fractale, la contamination métonymique du tableau vivant, l'articulation des mondes, par lesquelles nous entrons dans la pensée contemporaine des dispositifs.

\footnotetext{
${ }^{22}$ Lacan, Jacques: Le Séminaire, livre III, op. cit., p. 140.

${ }^{23}$ Lacan: op. cit., p. 314-315.
} 